\title{
TCF $7 L 2$ in the Go-DARTS study: evidence for a gene dose effect on both diabetes susceptibility and control of glucose levels
}

\author{
C. H. Kimber • A. S. F. Doney • E. R. Pearson • \\ M. I. McCarthy • A. T. Hattersley • G. P. Leese • \\ A. D. Morris • C. N. A. Palmer
}

Received: 17 October 2006 / Accepted: 19 February 2007 / Published online: 11 April 2007

(C) Springer-Verlag 2007

\begin{abstract}
Aims/hypothesis The gene encoding transcription factor 7like 2 (TCF7L2) has been identified as a type 2 diabetes locus from genome-wide linkage studies and subsequent association analysis. We investigated the role of two common variants in TCF7L2 in a large case-control study recruited from the Tayside region of Scotland, UK.

Subjects and methods We genotyped 6,516 participants for rs12255372 and rs7903146 and analysed the role in type 2 diabetes susceptibility using binary logistic regression. Age, sex and obesity status were examined as covariates. The
\end{abstract}

C. H. Kimber · C. N. A. Palmer

Population Pharmacogenetics Group,

Biomedical Research Centre,

Ninewells Hospital and Medical School,

Dundee, UK

A. S. F. Doney • E. R. Pearson · G. P. Leese - A. D. Morris

Diabetes Research Group, Division of Medicine and Therapeutics,

Ninewells Hospital and Medical School,

Dundee, UK

\section{I. McCarthy}

Oxford Centre for Diabetes, Endocrinology and Metabolism, Churchill Hospital Site,

Oxford, UK

\section{A. T. Hattersley}

Institute of Biomedical and Clinical Science,

Peninsula Medical School,

Exeter, UK

C. N. A. Palmer $(\bowtie)$

Biomedical Research Centre,

Ninewells Hospital and Medical School,

Dundee DD1 9SY, UK

e-mail: nuclear-receptor@dundee.ac.uk distribution of the genotypes within different treatment groups of cases was examined.

Results Both variants were associated with type 2 diabetes $\left(p<10^{-13}\right)$. The variants were present at very similar frequencies and were in strong linkage disequilibrium $\left(R^{2}=0.88, D^{\prime}=0.89\right)$. A gene dosage effect of the rare allele of both variants was observed, the heterozygote CT group of rs7903146 having an odds ratio of 1.36 (95\% CI 1.2-1.5, $p=1.54 \times 10^{-7}$ ) for type 2 diabetes and the TT homozygote having a greater risk $(\mathrm{OR}=2.03,95 \% \mathrm{CI} 1.7-2.5$, $\left.p=1.40 \times 10^{-12}\right)$. An interaction with sex was observed, the males displaying a higher degree of genotype-associated risk compared with the females $(p=0.023)$. The T allele was associated with increased $\mathrm{HbA}_{1 \mathrm{c}}$ levels in both cases and controls, and with decreased BMI and waist circumference in case but not controls. The T allele was overrepresented in individuals requiring insulin treatment and underrepresented in the patients being managed by diet alone $(p=0.006)$. Conclusions We have confirmed TCF7L2 to be a diabetes locus in a large case-control study in Tayside, UK. Our data suggest that variants of TCF7L2 may be associated with increased disease severity and therapeutic failure.

Keywords Body mass index · Disease severity · Genetics · Glucose control $\cdot \mathrm{HbA}_{1 \mathrm{c}} \cdot$ Insulin $\cdot$ Susceptibility

Transcription factor $\cdot$ Type 2 diabetes

\begin{tabular}{|c|c|}
\hline \multicolumn{2}{|c|}{ Abbreviations } \\
\hline Go-DARTS & Genetics of Diabetes Audit and Research \\
\hline OR & $\begin{array}{l}\text { Tayside Study } \\
\text { odds ratio }\end{array}$ \\
\hline PAR & population attributable risk \\
\hline SNP & single-nucleotide polymorphism \\
\hline
\end{tabular}




\section{Introduction}

The polygenic basis of type 2 diabetes has been intensely investigated by large consortia worldwide, and consensus on a range of valid susceptibility genes has emerged. Such genes, for example PPARG, KCNJ11 and CPN10, appear to contain common variation that confers only $10-20 \%$ additional risk of type 2 diabetes, and only studies with large numbers of case and controls have had sufficient statistical power to examine their role robustly [1]. Recently, Grant et al. investigated the role of variation in a region of chromosome 10 that had been implicated as a weak susceptibility locus by linkage analysis using wholegenome microsatellite markers in several studies [2]. Intensive single-nucleotide polymorphism (SNP) analysis and resequencing revealed that TCF 22 gene variants, including microsatellite DG10S478, SNP rs12255372 and rs7903146, are in tight linkage disequilibrium and were associated with type 2 diabetes [3]. This relationship was replicated in three studies providing robust statistical evidence for association with type 2 diabetes $\left(p<10^{-15}\right)$. This finding has now been replicated in several studies of both case-control and prospective study design [4-10]. Importantly, the risk alleles of TCF7L2 have also been shown to confer risk of conversion from impaired glucose tolerance to diabetes in the Diabetes Prevention Program [8]. In addition, the results of the Diabetes Prevention Program suggest that variation in this gene is associated with impaired beta cell function rather than insulin resistance.

In order to provide large-scale, population-based evidence for the role of this variant in type 2 diabetes, and to further characterise the role of these variants in the aetiology of diabetes, we have genotyped 6,516 individuals from Tayside, UK, who have been enrolled in the Wellcome Trust UK case-control study of type 2 diabetes. We have also examined the phenotypic and treatment profiles of the individuals with type 2 diabetes who harbour the risk variants.

\section{Subjects and methods}

Study population We studied type 2 diabetic patients and non-diabetic controls from the Wellcome Trust UK type 2 diabetes case-control collection (Go-DARTS2), which is a substudy of Diabetes Audit and Research Tayside (DARTS) [11-17]. All type 2 diabetes patients were physiciandiagnosed with type 2 diabetes and were recruited at primary or secondary care diabetes clinics, or invited to participate from primary care registers, and had not been characterised for GAD antibodies or MODY gene mutations. The controls were invited to participate through their primary care physicians or their workplace occupational health departments. None of the controls had a previous diagnosis of diabetes and their glucose tolerance status was unknown. All individuals in this ongoing study were recruited in Tayside between 1 October 2004 and 1 July 2006. All study participants were white. This study was approved by the Tayside Medical Ethics Committee and informed consent was obtained from all participants.

Genotyping We genotyped rs 12255372 and rs 7903146 by Taqman allelic discrimination assays (Applied Biosystems, Foster City, CA) using the following labelled probes and primers: rs 12255372 forward primer, TGCAAATCCAGCA GGTTAGCT; rs 12255372 reverse primer, GCAGAGGCC TGAGTAATTATCAGAA; probe 1, FAM-CCAGGAATAT CCAGGCAAGAATGACCA-BHQ-1; probe 2, YakimaYellow-CCCAGGAATATCCAGGCAAGAATTACCABHQ-1; rs7903146 forward primer, CCTCAAAACCTAG CACAGCTGTTAT; rs7903146 reverse primer, TGAAAA CTAAGGGTGCCTCATACG; probe 1, FAM-TAAGCAC TTTTTAGATATTATAT-MGB/NFQ; probe 2, VIC-CTAA GCACTTTTTAGATACTATAT-MGB/NFQ. The call rate for both assays was $\sim 98 \%$ and the duplicate concordance rate was $>99 \%$.

Statistical analysis Allele frequencies were calculated by gene counting. Hardy-Weinberg equilibrium was seen in both SNP distributions $(p>0.1)$. Binary logistic regression was used to compare the SNP frequencies in case and controls, and age, sex and obesity status were used as covariates. General linear modelling was used to compare quantitative parameters between disease status groups and between genotypic groups. All statistics were performed using SPSS v11 (SPSS Inc., Chicago, IL) for the Macintosh.

Table 1 Characteristics of study group

\begin{tabular}{llll}
\hline Characteristic & Controls & Cases & $p$ value \\
\hline Number of participants & 3,291 & 3,225 & \\
Sex (male/female) $(n)$ & $1,720 / 1,571$ & $1,821 / 1,404$ & $<0.001$ \\
Current smoker $(n)$ & 557 & 562 & 0.592 \\
Age (years) & $60.7 \pm 13.0$ & $65.3 \pm 11.2$ & $<0.001$ \\
BMI $\left(\mathrm{kg} / \mathrm{m}^{2}\right)$ & $26.8 \pm 4.6$ & $31.3 \pm 6.2$ & $<0.001$ \\
$\mathrm{HbA}_{1 \mathrm{c}}(\%)$ & $5.6 \pm 0.4$ & $7.7 \pm 1.5$ & $<0.001$ \\
Cholesterol $(\mathrm{mmol} / \mathrm{l})_{\text {Triacylglycerol }(\mathrm{mmol} / \mathrm{l})}$ & $5.3 \pm 1.1$ & $4.5 \pm 0.9$ & $<0.001$ \\
HDL-cholesterol $(\mathrm{mmol} / \mathrm{l})$ & $1.6 \pm 1.1$ & $2.2 \pm 1.4$ & $<0.001$ \\
LDL-cholesterol $(\mathrm{mmol} / \mathrm{l})$ & $3.0 \pm 1.0$ & $1.4 \pm 0.4$ & $<0.001$ \\
\hline
\end{tabular}

All values were determined from the clinical visit at study entry. Biochemical measures were obtained through the National Health Service clinical biochemistry system from blood taken at study entry. 
Table 2 TCF7L2 genotypes in the type 2 diabetes cases and controls

\begin{tabular}{|c|c|c|c|c|c|}
\hline rs2255372 genotype & Controls & Type 2 diabetes cases & rs7903146 genotype & Controls & Type 2 diabetes cases \\
\hline GG & $1,735(52.7)$ & $1,434(44.5)$ & $\mathrm{CC}$ & $1,714(52.1)$ & $1,405(43.7)$ \\
\hline GT & $1,323(40.2)$ & $1,432(44.4)$ & $\mathrm{CT}$ & $1,329(40.4)$ & $1,459(45.2)$ \\
\hline TT & $233(7.1)$ & $359(11.1)$ & TT & $248(7.5)$ & $361(11.2)$ \\
\hline Total & 3,291 & $\begin{array}{l}3,225 \\
p=2.05 \times 10^{-14}\end{array}$ & & 3,291 & $\begin{array}{l}3,225 \\
p=5.10 \times 10^{-14}\end{array}$ \\
\hline Minor allele frequency & 0.272 & 0.333 & & 0.277 & 0.338 \\
\hline
\end{tabular}

\section{Results}

This study involved a total of 6,516 individuals; 3,225 of these were cases who had been diagnosed with type 2 diabetes, and the remaining 3,291 individuals were population controls with no history of type 2 diabetes at the time of recruitment. The characteristics of the study population are shown in Table 1.

All individuals were typed for both rs12255372 and rs7903146 of TCF7L2 (Table 2). The allele frequencies of both SNPs in the controls were very similar to the reported frequencies in the Icelandic and Danish populations in the index studies, and the corresponding increased allele frequency was observed in the cases (Pearson $\chi^{2}$ for rs $12255372=58.48 ; p=2.05 \times 10^{-14} ;$ rs7903146=56.69; $\left.p=5.10 \times 10^{-14}\right)$. The increase for both SNPs was almost identical, tight linkage disequilibrium $\left(D^{\prime}=0.89, R^{2}=0.88\right)$ being observed. The high linkage disequilibrium in the Scottish population means that neither SNP provides significantly greater information than the other in this case-control comparison, although this may not be the case in other populations.

Logistic regression analysis was used to correct the association of rs7903146 and rs2255372 for age, sex and obesity status (Table 3 ). The susceptibility conferred by the risk alleles was clearly codominant, the rs7903146 heterozygote CT group having an odds ratio (OR) of $1.36(95 \%$ CI $1.2-1.5, p=1.54 \times 10^{-7}$ ) relative to the $\mathrm{CC}$ individuals, and the TT homozygote having a greater risk $(\mathrm{OR}=$ 2.03, 95\% CI 1.6-2.4; $p=1.40 \times 10^{-12}$ ) (Fig. 1). This represents an increased risk of type 2 diabetes in the TT individuals compared with the CT individuals $(\mathrm{OR}=1.48$, $95 \%$ CI $\left.1.2-1.8 ; p=9.89 \times 10^{-5}\right)$. The population attributable risk (PAR) observed for rs7903146 in this study was $18.9 \%$, in agreement with previous reports $[6,7]$.

Greater genotype-associated risk was observed in men compared with women, the odds ratios in the separate sexes being almost identical to those observed by Zhang et al. [6] (men, TT vs CC, OR=2.3, 95\% CI 1.8-3.1; $p=1.26 \times 10^{-10}$; women, TT vs $\mathrm{CC}, \mathrm{OR}=1.6,95 \% \mathrm{CI}$ $1.2-2.2 ; p=0.001)$. The additional power of our study, however, allowed detection of a significant interaction between genotype and sex $(p=0.023)$.

Linear regression was used to examine associations of rs7903146 with biochemical measures and adiposity in the controls and cases separately (Table 4 ). The results obtained with rs12255372 were very similar; the corresponding $p$ values $\left(p^{1}\right)$ are shown in Table 4 . No association was observed for either SNP with creatinine or any measure of dyslipidaemia in the cases or controls. In the cases, individuals with the $\mathrm{T}$ allele of rs7903146 were slimmer by $\mathrm{BMI}$ and waist circumference measures. $\mathrm{HbA}_{1 \mathrm{c}}$ was higher in the T-bearing individuals in both case and controls. BMI is a major determinant of $\mathrm{HbA}_{1 \mathrm{c}}$ in individuals with type 2 diabetes and we therefore included BMI as a covariate. There was a clear allelic association with $\mathrm{HbA}_{1 \mathrm{c}}$ in the cases and controls, indicating that the association between rs7903146 and $\mathrm{HbA}_{1 \mathrm{c}}$ is independent of BMI. This indicates that the T-allele carriers had increased $\mathrm{HbA}_{1 \mathrm{c}}$ despite being thinner. Individuals carrying

Table 3 TCF7L2 genotype is associated with type 2 diabetes after adjustment for age, sex and obesity in a binary logistic regression model

\begin{tabular}{|c|c|c|c|c|c|}
\hline rs 12255372 & OR $(95 \% \mathrm{CI})$ & $p$ value & rs7903146 & OR $(95 \% \mathrm{CI})$ & $p$ value \\
\hline GT & $1.35(1.20-1.51)$ & $2.80 \mathrm{e}^{-7}$ & $\mathrm{CT}$ & $1.36(1.21-1.52)$ & $1.54 \mathrm{e}^{-7}$ \\
\hline GG & $2.11(1.73-2.57)$ & $2.17 \mathrm{e}^{-13}$ & $\mathrm{TT}$ & $2.03(1.67-2.47)$ & $1.40 \mathrm{e}^{-12}$ \\
\hline Age (55-65 years) & $2.09(1.80-2.44)$ & $1.45 \mathrm{e}^{-21}$ & Age (55-65 years) & $2.09(1.88-2.53)$ & $2.93 \mathrm{e}^{-21}$ \\
\hline Age $(>65$ years $)$ & $3.31(2.88-3.80)$ & $3.10 \mathrm{e}^{-63}$ & Age (over 65 years) & $3.29(2.86-3.79)$ & $6.28 \mathrm{e}^{-63}$ \\
\hline Overweight & $2.29(1.98-2.64)$ & $1.27 \mathrm{e}^{-29}$ & Overweight & $2.28(1.97-2.63)$ & $2.74 \mathrm{e}^{-29}$ \\
\hline Obese & $9.42(8.08-10.97)$ & $2.45 \mathrm{e}^{-182}$ & Obese & $9.39(8.07-10.94)$ & $3.31 \mathrm{e}^{-182}$ \\
\hline Male & $1.21(1.09-1.36)$ & $4.27 \mathrm{e}^{-4}$ & Male & $1.22(1.09-1.36)$ & $4.27 \mathrm{e}^{-4}$ \\
\hline
\end{tabular}

Obese, BMI $\geq 30 \mathrm{~kg} / \mathrm{m}^{2}$ and above; overweight, $25-30 \mathrm{~kg} / \mathrm{m}^{2}$

Referent categories: rs7903146 C/C; age under 55 years; individuals with BMI $<25 \mathrm{~kg} / \mathrm{m}^{2}$ 


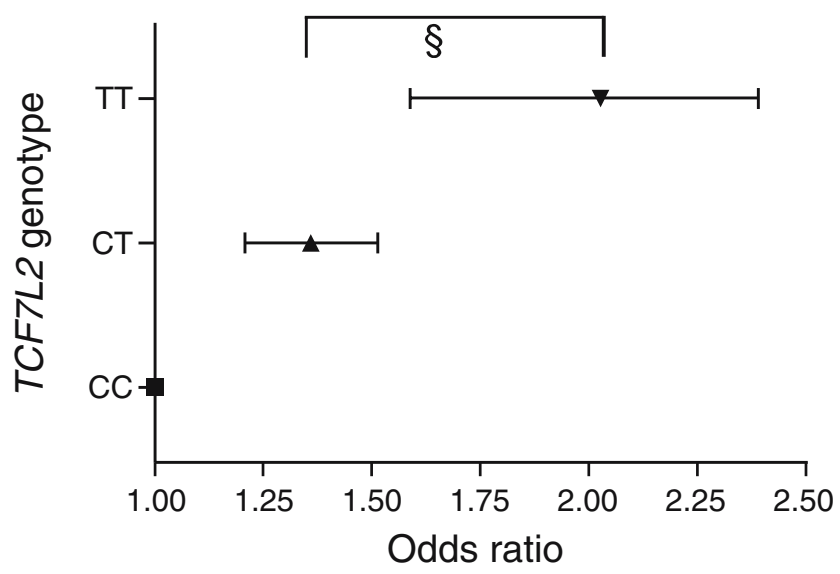

Fig. 1 TCF7L2 genotype demonstrates a clear codominant association with type 2 diabetes. Shown are the point estimates of the odds ratios for rs7903146 CT and TT vs the CC referent genotype. Error bars are $95 \%$ confidence intervals. ${ }^{\S} p=9.89 \times 10^{-5}$

the $\mathrm{T}$ allele of rs7903146 were also diagnosed slightly younger than the $\mathrm{CC}$ individuals (1.4 years difference between $\mathrm{CC}$ and TT; $p=0.031$ ). As an additional measure of disease severity and therapeutic control, we examined the distribution of rs7903146 alleles within the three major treatment modalities for type 2 diabetes, i.e. diet alone, oral hypoglycaemics and insulin treatment (Fig. 2). This demonstrates that the individuals bearing the $\mathrm{T}$ allele of rs7903146 were less likely to have been managed on diet alone, and were more likely to have received insulin treatment $\left(\chi^{2}\right.$ test for trend, $\left.p=0.007\right)$, and this remained significant after adjustment for age, sex, BMI and smoking status $(p=0.006)$. The odds ratio for rs7903146 TT homozygotes for insulin treatment vs non-insulin treatment was $1.32(95 \%$ CI $1.02-1.7 ; p=0.038)$ relative to the common CC homozygote. In addition, the association of

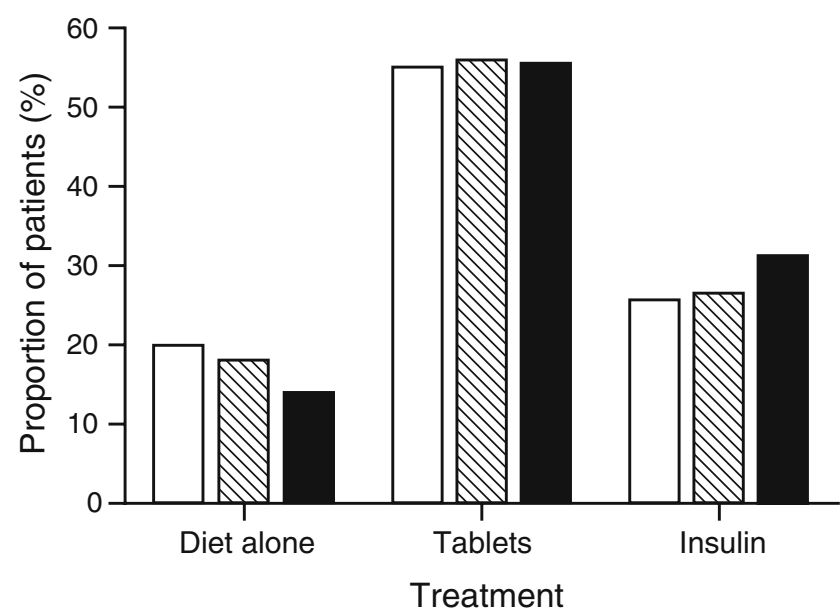

Fig. 2 Association of treatments for the diabetic patients and their genotypes. Shown is the proportion of each genotype group of rs7903146 in each treatment class: CC (open bars); CT (hatched bars); TT (black bars). Tablets group constitutes individuals on oral hypoglycaemic agents without insulin. Insulin group contains insulin monotherapy and combined insulin/oral hypoglycaemic agent treated individuals. Similar results were obtained with rs $12255372 . \chi^{2}$ test for trend $=7.37, p=0.007$

the TT homozygote with active medication vs diet alone was significant $(\mathrm{OR}=1.55,95 \%$ CI $1.12-2.16 ; p=0.01)$.

\section{Discussion}

This study provides evidence of the robust nature of the association between common variation in the TCF7L2 gene and type 2 diabetes. This association has been more readily replicated than the other previous candidate gene variants as the allele frequency of the variant is more than $30 \%$ and

Table 4 Characteristics of control and case populations by $T C F 7 L 2$ genotype

\begin{tabular}{|c|c|c|c|c|c|c|c|c|c|c|}
\hline \multirow[b]{2}{*}{ Rs7903146 } & \multicolumn{4}{|l|}{ Controls } & \multicolumn{6}{|l|}{ Cases } \\
\hline & $\mathrm{CC}$ & $\mathrm{CT}$ & $\mathrm{TT}$ & $p$ value $^{\mathrm{a}}$ & $p$ value $^{\mathrm{c}}$ & $\mathrm{CC}$ & $\mathrm{CT}$ & TT & $p$ value $^{\mathrm{a}}$ & $p$ value $^{\mathrm{c}}$ \\
\hline BMI $\left(\mathrm{kg} / \mathrm{m}^{2}\right)$ & $26.9(4.5)$ & $26.8(4.6)$ & $26.7(4.7)$ & 0.802 & 0.507 & $31.6(6.3)$ & $31.3(6.0)$ & $30.4(6.3)$ & $0.002^{\mathrm{b}}$ & $0.008^{\mathrm{b}}$ \\
\hline Waist $(\mathrm{cm})$ & $92.9(13.1)$ & $92.5(13.0)$ & $91.7(13.4)$ & 0.549 & 0.334 & $104.8(14.3)$ & $104.3(13.6)$ & $102.1(14.5)$ & $0.001^{\mathrm{b}}$ & $0.002^{\mathrm{b}}$ \\
\hline $\mathrm{HbA}_{1 \mathrm{c}}(\%)$ & $5.56(0.4)$ & $5.60(0.4)$ & $5.63(0.5)$ & $0.003^{\mathrm{b}, \mathrm{d}}$ & $0.13^{\mathrm{b}}$ & $7.64(1.5)$ & $7.72(1.5)$ & $7.88(1.4)$ & $0.012^{\mathrm{b}, \mathrm{d}}$ & $0.002^{\mathrm{b}}$ \\
\hline Cholesterol (mmol/l) & $5.32(1.1)$ & $5.35(1.1)$ & $5.25(1.0)$ & 0.375 & 0.480 & $4.48(0.9)$ & $4.45(0.9)$ & $4.46(0.9)$ & 0.757 & 0.354 \\
\hline HDL-cholesterol (mmol/l) & $1.65(0.5)$ & $1.64(0.5)$ & $1.66(0.5)$ & 0.563 & 0.730 & $1.37(0.4)$ & $1.37(0.4)$ & $1.39(0.4)$ & 0.306 & 0.346 \\
\hline LDL-cholesterol (mmol/l) & $2.98(1.0)$ & $2.98(1.0)$ & $2.92(0.9)$ & 0.632 & 0.852 & $2.17(0.8)$ & $2.12(0.8)$ & $2.14(0.8)$ & 0.393 & $0.024^{\mathrm{b}}$ \\
\hline Triacylglycerol (mmol/l) & $1.56(1.0)$ & $1.64(1.3)$ & $1.56(1.0)$ & 0.326 & 0.406 & $2.21(1.5)$ & $2.25(1.4)$ & $2.21(1.4)$ & 0.756 & 0.225 \\
\hline Serum creatinine $(\mu \mathrm{mol} / \mathrm{l})$ & $94.5(19.4)$ & $95.2(18.4)$ & $93.3(19.5)$ & 0.419 & 0.277 & $98.8(24.1)$ & $99.8(27.0)$ & $97.5(23.4)$ & 0.252 & 0.834 \\
\hline Age at diagnosis (years) & & & & & & $57.5(11.4)$ & $57.1(11.5)$ & $56.1(12.1)$ & $0.031^{\mathrm{b}, \mathrm{e}}$ & 0.114 \\
\hline
\end{tabular}

BMI was included as a covariate in the analysis of $\mathrm{HbA}_{1 \mathrm{c}}$.

${ }^{a}$ General linear model analysis with age at recruitment, sex and smoking status as covariates

${ }^{\mathrm{b}}$ Nominally significant $p$ values at $p<0.05$

${ }^{\mathrm{c}}$ Corresponding $p$ value for rs 12255372

${ }^{\mathrm{d}} p=0.001$ overall adjusted for case-control status

${ }^{\mathrm{e}}$ Not significant after Bonferroni correction for multiple testing 
a clear gene dose effect has been observed, the homozygote rare variant conferring an odds ratio of about 2.0. This has meant, for the first time, that studies containing hundreds, rather than thousands, of cases have had sufficient statistical power to detect this effect reliably. The possibility of this result arising from population stratification was minimised by the use of a relatively homogeneous casecontrol population of white individuals from a single region of Scotland, and is highly unlikely given the global consistency of the association [18-21] and direct testing of stratification in a whole-genome association study [22]. The use of more than 6,000 individuals in this study has provided the power to separate the three genotypes statistically, the rare homozygote group bearing significantly greater risk than the CT heterozygotes, and has provided power to show that males have a greater genotypeassociated risk than females. These data are in agreement with the previous case-control studies and confirm that the lack of gene dosage effects seen in prospective studies is likely to have been due to lack of power. Using binary logistic modelling, we have shown that the association is robust to adjustment for obesity status, sex and age group, the odds ratios for the $\mathrm{T}$ homozygotes being similar to those for important conventional risk factors, such as being overweight or middle-aged.

In addition, we also confirm recent studies that suggested that the variants did not have a primary effect on adiposity but were associated with reduced BMI in cases with type 2 diabetes only [7]. This would suggest that the variants predispose individuals to type 2 diabetes at a lower level of adiposity. The mechanism for this may be impaired pancreatic beta cell function, but detailed molecular mechanisms for this are not yet clear. Also, in agreement with earlier studies, we found that the T allele of rs7903146 was associated with a slightly earlier age of diagnosis, and although the association does not stand up to correction for multiple testing within this study, it does provide replication support for earlier observations $[9,23]$ and is consistent with the observation that the risk alleles of TCF7L2 are enriched further in groups with early-onset type 2 diabetes [7]. We have extended these observations by demonstrating a relationship with disease severity and therapeutic status. The rs7903146 $\mathrm{T}$ allele is associated with higher $\mathrm{HbA}_{1 \mathrm{c}}$ in both controls and individuals with type 2 diabetes, and although the individual observations in case and controls do not withstand correction for multiple testing, the overall association between rs7903146 is highly significant when adjusted for case-control status ( $p=0.001$ ), and this measure is still significant after Bonferroni correction. The association with higher $\mathrm{HbA}_{1 \mathrm{c}}$ in the T-allele carriers with type 2 diabetes is evident despite the more intensive treatment observed in T-allele carriers, and reinforces the notion that the $\mathrm{T}$ allele may be associated with greater disease severity.
Prospective studies have demonstrated the role of TCF7L2 variants in the rate of progression from impaired glucose tolerance to diabetes. Longitudinal studies are also now required to confirm our findings that, even in the diabetic state, this gene may be associated with disease that is managed poorly with standard therapies. Further studies are required to determine the suitability of these individuals for particular oral hypoglycaemic agents. Although the discovery of TCF7L2 as a major predisposition gene for type 2 diabetes does not immediately inform patient care [24], it is hoped that careful dissection of the performance of current therapies in patients with different TCF7L2 genotypes may lead to specific treatment strategies based on TCF $7 L 2$ genotype.

Acknowledgements We would like to thank all the participants in this study and the team of nurses who have made this study possible. The UK type 2 diabetes case-control collection (Go-DARTS2) was funded by the Wellcome Trust. C. N. A. Palmer and A. D. Morris are supported by the Scottish Executive Chief Scientist's Office as part of the Generation Scotland initiative. E. R. Pearson holds a NHS Education for Scotland Clinician Scientist fellowship.

Duality of interest None of the authors has any conflict of interest.

\section{References}

1. McCarthy MI, Zeggini E (2006) Genetics of type 2 diabetes. Curr Diab Rep 6:147-154

2. Reynisdottir I, Thorleifsson G, Benediktsson R et al (2003) Localization of a susceptibility gene for type 2 diabetes to chromosome 5q34-q35.2. Am J Hum Genet 73:323-335

3. Grant SF, Thorleifsson G, Reynisdottir I et al (2006) Variant of transcription factor 7-like 2 (TCF7L2) gene confers risk of type 2 diabetes. Nat Genet 38:320-323

4. Damcott CM, Pollin TI, Reinhart LJ et al (2006) Polymorphisms in the transcription factor 7-like 2 (TCF7L2) gene are associated with type 2 diabetes in the Amish: replication and evidence for a role in both insulin secretion and insulin resistance. Diabetes 55:2654-2659

5. Scott LJ, Bonnycastle LL, Willer CJ et al (2006) Association of transcription factor 7-like 2 (TCF7L2) variants with type 2 diabetes in a Finnish sample. Diabetes 55:2649-2653

6. Zhang C, Qi L, Hunter DJ et al (2006) Variant of transcription factor 7-like 2 (TCF7L2) gene and the risk of type 2 diabetes in large cohorts of U.S. women and men. Diabetes 55:2645-2648

7. Groves CJ, Zeggini E, Minton J et al (2006) Association analysis of 6,736 U.K. subjects provides replication and confirms TCF7L2 as a type 2 diabetes susceptibility gene with a substantial effect on individual risk. Diabetes 55:2640-2644

8. Florez JC, Jablonski KA, Bayley N et al (2006) TCF7L2 polymorphisms and progression to diabetes in the Diabetes Prevention Program. N Engl J Med 355:241-250

9. Cauchi S, Meyre D, Dina C et al (2006) Transcription factor TCF7L2 genetic study in the french population: expression in human beta-cells and adipose tissue and strong association with type 2 diabetes. Diabetes 55:2903-2908

10. Saxena R, Gianniny L, Burtt NP et al (2006) Common single nucleotide polymorphisms in TCF7L2 are reproducibly associated 
with type 2 diabetes and reduce the insulin response to glucose in nondiabetic individuals. Diabetes 55:2890-2895

11. Doney A, Fischer B, Frew D et al (2002) Haplotype analysis of the PPARgamma Pro12Ala and C1431T variants reveals opposing associations with body weight. BMC Genet 3:21

12. Doney AS, Fischer B, Cecil JE et al (2004) Association of the Pro12Ala and C1431T variants of PPARG and their haplotypes with susceptibility to Type 2 diabetes. Diabetologia 47:555-558

13. Doney AS, Fischer B, Cecil JE et al (2003) Male preponderance in early diagnosed type 2 diabetes is associated with the ARE insertion/deletion polymorphism in the PPP1R3A locus. BMC Genet 4:11

14. Doney AS, Fischer B, Lee SP, Morris AD, Leese G, Palmer CN (2005) Association of common variation in the PPARA gene with incident myocardial infarction in individuals with type 2 diabetes: a Go-DARTS study. Nucl Recept 3:4

15. Doney AS, Fischer B, Leese G, Morris AD, Palmer CN (2004) Cardiovascular risk in type 2 diabetes is associated with variation at the PPARG locus: a Go-DARTS study. Arterioscler Thromb Vasc Biol 24:2403-2407

16. Doney AS, Lee S, Leese GP, Morris AD, Palmer CN (2005) Increased cardiovascular morbidity and mortality in type 2 diabetes is associated with the glutathione $\mathrm{S}$ transferase theta-null genotype: a Go-DARTS study. Circulation 111:2927-2934

17. Morris AD, Boyle DI, MacAlpine R et al (1997) The diabetes audit and research in Tayside Scotland (DARTS) study: electronic record linkage to create a diabetes register. DARTS/MEMO Collaboration. Br Med J 315:524-528

18. Helgason A, Palsson S, Thorleifsson G et al (2007) Refining the impact of TCF7L2 gene variants on type 2 diabetes and adaptive evolution. Nat Genet 39:218-225

19. Chandak GR, Janipalli CS, Bhaskar S et al (2007) Common variants in the TCF7L2 gene are strongly associated with type 2 diabetes mellitus in the Indian population. Diabetologia 50:63-67

20. Humphries SE, Gable D, Cooper JA et al (2006) Common variants in the TCF7L2 gene and predisposition to type 2 diabetes in UK European Whites, Indian Asians and Afro-Caribbean men and women. J Mol Med 84(12 Suppl):1-10

21. van Vliet-Ostaptchouk JV, Shiri-Sverdlov R, Zhernakova A et al (2007) Association of variants of transcription factor 7-like 2 (TCF7L2) with susceptibility to type 2 diabetes in the Dutch Breda cohort. Diabetologia 50:59-62

22. Sladek, R, Rocheleau G, Rung J et al (2007) A genome-wide association study identifies novel risk loci for type 2 diabetes. Nature 445:881-885

23. Lehman DM, Hunt KJ, Leach RJ et al (2007) Haplotypes of transcription factor 7-like 2 (TCF7L2) gene and its upstream region are associated with type 2 diabetes and age of onset in Mexican Americans. Diabetes 56:389-393

24. Janssens AC, Gwinn M, Valdez R, Narayan KM, Khoury MJ (2006) Predictive genetic testing for type 2 diabetes. BMJ 333:509-510 